\title{
3D goes mainstream
}

\author{
Could 2010 be the year that 3D display technology takes off in cinemas and homes? All the signs \\ suggest that the answer is yes.
}

By the time you read this Editorial, Avatar - the latest film from the famous Canadian director James Cameron - will have hit 3D cinemas around the world. Some within the industry are likening the impact of this event (along with the flood of other upcoming 3D feature films) to the introduction of colour film. Others believe that it marks the successful transition of $3 \mathrm{D}$ cinema from children's animations to mainstream films. This technology will also be making its way into homes, with 3D-enabled televisions and cameras now reaching the marketplace. After decades of research, this technology finally seems set to be welcomed by the general public.

In the next few years, over 50 movies from filmmakers such as Steven Spielberg, Peter Jackson, George Lucas and

Robert Zemeckis are scheduled for cinema release in RealD 3D, a popular commercial stereoscopic projection system for $3 \mathrm{D}$ digital cinema.

The modern 3D experience has come a long way since its early trials two decades ago. It is flicker-free, there is no need to hold your head upright and the paper glasses are now replaced with lightweight, reusable and recyclable plastic 'shades'. Designer brands and prescription versions of these glasses will even be available soon, allowing movie fans to have their own personalized pair. This demonstrates just how mainstream $3 \mathrm{D}$ is expected to become.

This momentum of development is not going unnoticed by consumer electronics manufacturers, who have their sights firmly set on introducing 3D displays to the home. It is not difficult to imagine why home viewers are anticipating this; for example, it will allow sports fans to see bumps on a golf green and envisage the same putting line as that of the player. Meanwhile, the massive computer games market is waiting anxiously for high-performance yet costeffective solutions to arrive. With the world's largest consumer electrons show CES 2010 (Las Vegas, 7-10 Jan 2010) just around the corner, media reports are already anticipating a strong showing of the latest $3 \mathrm{D}$ television technology about to come to market.

Indeed, Nature Photonics has already had a sneak preview at the International

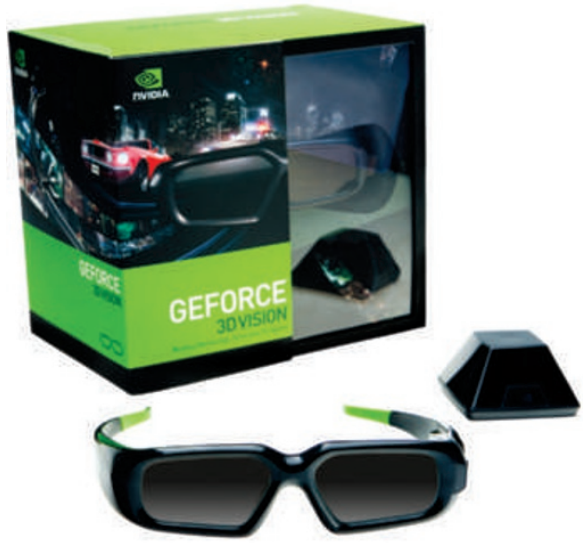

enthusiastic about the technology), have now announced notebook computers that contain the graphics chips required to achieve a 3D display.

JVC presented the GD-463D10, a display that achieves $3 \mathrm{D}$ vision by utilizing a circularly polarized filter system. A filter switches the polarization of the display's output many times per second, with lefthanded polarization corresponding to the image for one eye and the right-handed polarization for the other. Hence, passive glasses with normal circular polarization filters are all that is required for this scheme. Panasonic has also developed a 50-inch fully high-definition 3D plasma display system, which is based on the active shuttering glasses described above and is due to launch in 2010. Taiwan, a hotbed of display makers, is also having a strong presence in the $3 \mathrm{D}$ display marketplace. AU Optronics Corporation has unveiled a 65 -inch 3D display, and Chi Mei Optoelectronics has presented a high-definition $120 \mathrm{~Hz}$ 3D panel that uses shutter-type glasses.

Devices for capturing images in 3D are also coming to market. A digital camera from FujiFilm - the FinePix REAL 3D System - has been on sale in Japan since August 2009. The camera captures images through a pair of lenses spaced about $8 \mathrm{~cm}$ apart and instantaneously synthesizes them into a stereoscopic image. Fujifilm has also developed viewing and printing technology to allow consumers to enjoy the resulting images.

What kind of 3D technology will come to fruition in the future? Kevin Thompson of Optical Research Associates believes that applications are pointing towards head-worn displays, based on his work with Jannick Rolland of the University of Rochester's Institute of Optics. The idea is to not only to display images, but also to augment reality with desirable information and features, for aiding surgery or overlaying path maps on mountains, for example.

From all this activity, it certainly seems that there is now a big push to make 3D technology a commercial reality. The big question is whether consumers will readily embrace it. 